\title{
Extraction of Drift Direction and Speed of Sea Ice of the Huanghai and Bohai Sea Based on GOCI Satellite
}

\author{
Shengwen Gong ${ }^{1, a}$, Zhuo Chen $^{2, b}$ and Xi Zhang ${ }^{3, c}$ \\ ${ }^{1,2}$ Qingdao University of Science \& Technology, SongLing Rd. 99, 266071 Qingdao, China \\ ${ }^{3}$ First Institute of Oceanography, SOA, XianxiaLing Rd. 6,266071 Qingdao, China \\ aqd_gsw@163.com, bchenzhuo_qust@163.com, 'xi.zhang@fio.org.cn
}

\begin{abstract}
Keywords: Sea Ice Monitoring; Satellite Remote Sensing; Harris Algorithm; SIFT Algorithm; Feature Extraction; Image Matching
\end{abstract}

\begin{abstract}
At present, GOCI satellite is the only means to monitor the drift of sea ice in a wide range. The high temporal resolution makes it fit for monitoring Marine phenomenon and process in time series. It improves the possibility of obtaining a high quality image in a short period of time. GOCI satellite is with great applied potentiality and value. In order to use the information of GOCI satellite image effectively, Harris algorithm and SIFT algorithm are combined to extract the feature points of image of sea ice and match images. Extraction of drift direction and speed of sea ice of the Huanghai and Bohai Sea is realized in this paper.
\end{abstract}

\section{Introduction}

Marine disasters in the Huanghai and Bohai Sea region are with the characteristic of multiple kinds, high frequency, and strong emergency. Sea ice is one of the mainly disasters in the Huanghai and Bohai Sea region, and it is one of most important factors which influences shipping, the drilling construction and production in ice area. The drift of floating ice is a huge threat to the Marine exploitation, production and shipping in winter. With the development of the Marine economy, the loss caused by sea ice disaster is increasing. So the influence of sea ice must be paid more attention when moving in the sea ice area. The common problem of current sea ice monitoring methods is that the corresponding equipment needs to be installed, the requirement on manpower and material resources is high, and the measuring range is so small that the monitoring results of drift of sea ice in a large area can not be gotten. Since the poor work environment of sea in the cold area, the manner to monitor drifting direction and speed of sea ice by satellite remote sensing is practically significant for sea ice forecast, ocean engineering and so on.

GOCI satellite with high spatial resolution and high temporal resolution can cover part of Bohai, Huanghai and Donghai Sea. Compared with SeaWiFS, MODIS and other international mainstream color satellite, the characteristic of GOCI is that the 8 scene data of every daytime which is 1 scene every hour can be provided. So GOCI satellite satisfies the need of monitoring drift of sea ice and provides real-time and high frequency data. The real-time and accurate monitoring information of sea ice can be provided to decrease the loss caused by disaster.

\section{Extraction of feature points of ice}

Feature extraction method generally includes two stages. The first one is image segmentation. Extract the target area or its border and extract the basic characteristics such as gray scale, texture and shape description of the target. The second is finding or construction feature invariant of target image which is invariant when target move in no matter what kind. When the number of extracted feature points is too much, there will be large amounts of interference information such as false matching points. The accuracy and speed of matching will be affected by the interference information. When there is small number of extracted feature points, it will make bad effect on precise matching. The 
method of feature points matching needs a lot of efficient points in the two image point sets. The complexity of algorithm is bigger when the number of image feature points is more.

Harris characteristic angular point monitoring. In the current image matching manners, the common extraction algorithm of angular point based on gray level mainly includes Moravec algorithm, SUSAN algorithm and Harris algorithm, etc. Schmid compares the repeat rate and local area around angular point of Moravec, SUSAN and Harris algorithms. The experimental results show that Moravec and SUSAN algorithms are with more false detection and leakage detection, and can not match the angular points in the images before and after the rotation. Harris characteristic angular point monitoring can get better result. So the Harris algorithm is used to monitor feature points of sea ice.

SIFT extraction algorithm of feature points. Lowe proposed a kind of detection method of scale invariant key-point based on the property of scale space. The extremum point both in scale domain and space domain is as a key point. The key point is set as the center of characteristics region, at the same time the scale which the key points lie in is used to determine the size of the feature area. This method can solve the problem of location and size of characteristics region better at the same time. The feature points extracted by SIFT extraction method of local invariant feature points have better robustness to the image scale, rotation, light and changes of noise. But the algorithm adopts 128 dimensional vectors to describe the extracted feature points, so the speed is slow. Secondly, the algorithm is based on local area description which requires enough textures.

\section{Feature point matching algorithm of sea ice}

The scheme to obtain preliminary feature points in the image is to use the Harris extraction algorithm, and then SIFT algorithm is applied to select again from these feature points. The scheme is equal to use two kinds of feature point extraction algorithm to image processing.

\section{Drift information extraction of sea ice}

In general, image changes such as scale zooming, translation and rotation exist in the process of drift of sea ice. For this reality, the extraction of its information is carried out by the SIFT feature points extraction algorithm based on two sea ice images in a certain time interval within the scope of a certain latitude and longitude. Then execute image matching. The drift velocity is concluded by drawing the drift trajectory of sea ice, calculating displacement according to drift trajectory based on the known time interval.

Extraction of drift direction of sea ice. Extraction of drift direction of sea ice is equivalent to drawing the drift trajectory of sea ice. The specific implementation steps are as follows:

1) Obtain two sea ice images of Huanghai and Bohai Sea in a certain time interval, within the scope of the same latitude and longitude.

2) Apply improved SIFT algorithm to extract the feature points of the two sea ice images respectively.

3) Calculate the matched points by applying the improved matching algorithm based on the nearest neighbor principle of Euclidean distance.

4) Show the drift trajectory of sea ice in the interval on the recent sea ice images, which contains start points and end points, as well as the general trajectory.

5) Draw the trajectories which can be considered as the general direction of drift in the interval time.

Extraction of drift speed of sea ice. The specific implementation steps of speed extraction of sea ice drift based on the drift trajectories are as follows.

1) Obtain the shooting range of sea ice images which is the range of longitude and latitude.

2) Construct the corresponding relationship between image pixels and latitude and longitude position, and construct a transformation formula which can be used to calculate from any point in the image to the latitude and longitude coordinates at sea. 
3) Find the coordinates of the start point of any trajectory in the image, and converts it to corresponding coordinate at sea. Apply distance calculation formula on latitude and longitude coordinates to calculate the displacement.

4) Calculate the drift velocity of sea ice on the trajectory by combining the known time interval.

Experiment test. The experiment platform is MATLAB 7.1.

The data sources are two sea ice images of Huanghai and Bohai Sea based on GOCI satellite. Shooting time interval is 1 hour.

The original figure is as shown in Figure 1. The result is shown in Figure 2.

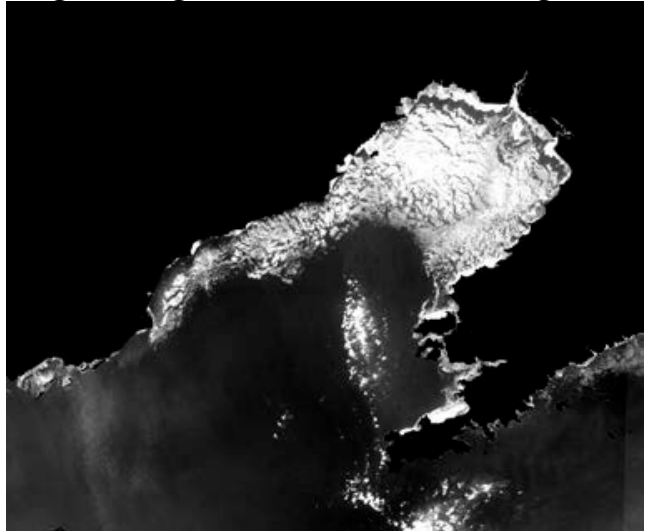

(a) Original Figure 1

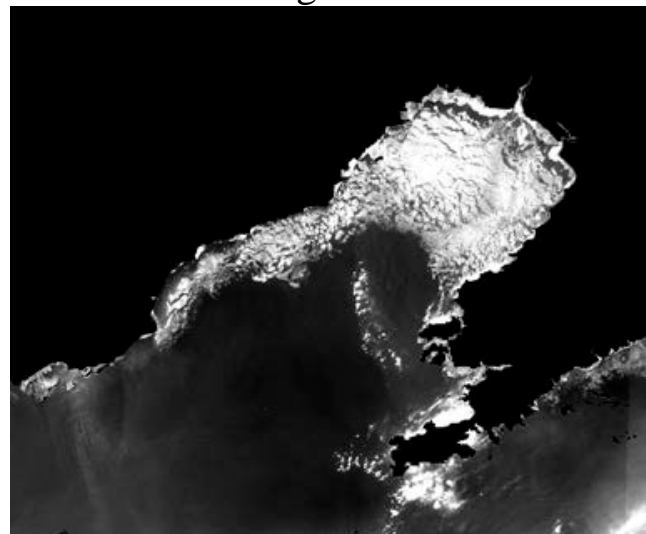

(b) Original Figure 2

Fig. 1 Original Huang Bohai sea ice images based on the GOCI satellite

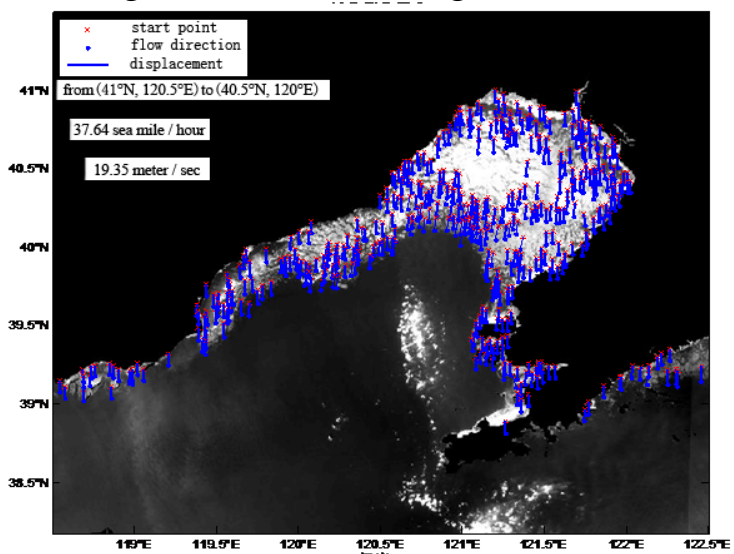

Fig. 2 Experiment result on Huang Bohai sea ice images based on GOCI satellite

\section{Conclusions}

The Harris algorithm and SIFT algorithm are combined based on some existing feature points extraction algorithms and matching methods. The sea ice images of Huanghai and Bohai Sea based on GOCI satellite are as research object. The extraction of drifting direction and speed of sea ice is completed.

\section{Acknowledgements}

The authors thank the Korea Ocean Satellite Center for providing the GOCI data.This work was financially supported by the Open fund project of Shandong Provincial Key Laboratory of Marine Ecological Environment and Disaster Prevention and Mitigation (2012006), science and technology plan project of the colleges and universities in Shandong province (J14LN74).

\section{References}

[1] David G Lowe. Object recognition from local scale-Invariant features[C]. In: Proceeding of the International Conference on Computer Vision (ICCV'1999), 1999.20 27. 
[2] David G Lowe. Distinctive image features from scale-invariant keypoints[J]. International Journal of Computer Vision,2004,60(2): 91 110.

[3] Scharfen G. and S. Khalsa. Assessing the Utility of MODIS for Monitoring Snow and Sea Ice Externt. Proceedings of Earsel-lissing-workshop Observing our Cryosphere from Space, 2002,11(13) :122 127.

[4] Drue, C., and G. Heinemann. High-resolution maps of the sea-ice concentration from MODIS satellite data. Geophys Res. Lett., 2004,31:L20403 1-5.

[5]Xie Feng, Gu Wei, Yuan Yi and Chen Yunhao. ESTIMATION OF SEA ICE RESOURCES IN LIAODONG GULF USING REMOTE SENSING [J]. RESOURCES SCIENCE, 2003,25(3): 17 23.

[6]Dorko G.,Schmid C. Selection of Scale Invariant Neighborhoods for Object Class Recognition[C]. In: Proceedings International Conference on Computer Vision,2003.634 640. 